\title{
Avaliação do estudante na educação remota (ER) e à distância (EAD): como desenvolver de modo efetivo, enfatizando a devolutiva
}

\author{
Student Assessment in Remote and Distance Education: how to practice \\ effectively emphasizing feedback.
}

Fábio Antônio Perecim Volpe ${ }^{1}$ (D), Silvana Maria Quintana² (D), Marcos de Carvalho Borges ${ }^{3}$ (D), Luiz Ernesto de Almeida Troncon ${ }^{4}$ (D)

\begin{abstract}
RESUMO
A avaliação do estudante, que constitui parte essencial da sua educação, sobretudo nas profissões da saúde, vem sofrendo grandes avanços nos seus conceitos fundamentais e, sobretudo, na sua prática cotidiana. Boa parte dessas mudanças relaciona-se à crescente utilização de estratégias de ensino e aprendizagem mediadas pela tecnologia da informação e comunicação (educação à distância ou ensino remoto), que foi, mais recentemente, acelerada pela pandemia do COVID-19. Deve ser também destacado que parte dessas mudanças implicou em maior reconhecimento da grande efetividade da avaliação formativa, desenvolvida, sobretudo, por meio da prática da devolutiva (feedback) frequente e de boa qualidade, seguindo recomendações técnicas adequadas. Neste artigo, apresentam-se alguns conceitos básicos de avaliação educacional e da avaliação do estudante, discute-se o papel estratégico da avaliação formativa e o poder da devolutiva (feedback), descrevem-se algumas das características da devolutiva efetiva e as correspondentes recomendações para a sua prática e descreve-se brevemente um conjunto de sugestões sobre possíveis maneiras efetivas de praticar a avaliação do estudante na educação remota e à distância, com ênfase na finalidade formativa, mas sem desconsiderar a somativa, que implica na tomada de decisões como aprovação ou reprovação.
\end{abstract}

Palavras-chave: Avaliação educacional; Avaliação do estudante; Avaliação formativa; Educação profissional; Educação à distância, Covid-19.

\begin{abstract}
Student assessment comprises an essential part of his or her education and professional training, especially in the health professions. Fundamental concepts on student assessment and their implications in school routinely practice has been evolving rapidly in recent times. Much of these changes are consequences of the increased utilization of teaching and learning strategies mediated by information and communication technology (distance education and online learning), which has been more recently accelerated by the COVID-19 pandemic. An important aspect of these changes implies in greater recognition of the educational power and effectiveness of formative assessment, carried out by frequent, high-quality feedback to students on their learning achievements, following appropriate technical recommendations. In this article, we present some basic concepts of educational evaluation and student assessment and discuss the strategic role of formative assessment and the power of feedback. We also describe some of the characteristics of effective feedback and the corresponding recommendations, and present suggestions on possible effective ways to practice student assessment in the context of remote teaching and distance education, with an emphasis on the formative purpose, but not disregarding summative assessment leading to pass or fail decision-making.
\end{abstract}

KEYWORDS: Educational assessment; Educational measurement; Distance education; Online learning, Covid-19.

Universidade de São Paulo. Faculdade de Medicina de Ribeirão Preto, Divisão de Cirurgia Pediátrica, Departamento de Cirurgia e Anatomia, Centro de Desenvolvimento Docente para o Ensino. Membro do Centro de Desenvolvimento Docente para o Ensino.

2 Universidade de São Paulo. Faculdade de Medicina de Ribeirão Preto, Departamento de Ginecologia e Obstetrícia, Setor de Obstetrícia, Centro de Desenvolvimento Docente para o Ensino. Membro do Centro de Desenvolvimento Docente para o Ensino.

3 Universidade de São Paulo. Faculdade de Medicina de Ribeirão Preto, Divisão de Emergências Clínicas, Departamento de Clínica Médica. Membro do Centro de Desenvolvimento Docente para o Ensino.

4 Universidade de São Paulo. Faculdade de Medicina de Ribeirão Preto, Divisão de Gastroenterologia, Departamento de Clínica Médica. Membro do Centro de Desenvolvimento Docente para o Ensino.

https://doi.org/10.11606/issn.2176-7262.rmrp.2021.184773 


\section{MENSAGENS IMPORTANTES:}

1. O processo de avaliação, de acordo com sua finalidade, pode ser classificado em somativo (para decisões de progresso e classificação do estudante), formativo (para fomentar o aprendizado) e diagnóstico (para inferir sobre a qualidade do programa educacional).

2. O feedback ou devolutiva é parte fundamental do processo de avaliação formativa e, se aplicado de maneira intencional e sistematizada, produz efeitos positivos na aprendizagem, estimula a reflexão e orienta o estudante para direcionar seus esforços em pontos de melhoria específicos.

3. No ambiente virtual de ensino o professor encontra várias oportunidades de implantar avaliações formativas, utilizando recursos como o e-portfolio, envio de relatórios de atividades, tarefas, perguntas e respostas (quizzes), fóruns de discussão e chats para, por exemplo, propor resoluções de problemas. As devolutivas podem acontecer em modo síncrono ou em devolutivas eletrônicas padronizadas na própria plataforma de ensino e aprendizagem.

4. 4. O cenário da pandemia da Covid-19 e a necessidade de avaliar o estudante em ensino remoto emergencial, contribuiu significativamente para o reconhecimento e prática de formas de avaliação formativa para além do apontamento de necessidade de melhorias, mas também como uma alternativa de reunir informações de progresso do estudante dentro das unidades de ensino.

\section{INTRODUÇÃO}

A avaliação do estudante constitui parte essencial da sua educação ${ }^{1,2}$. Nas últimas décadas ocorreram grandes mudanças nos conceitos relacionados à educação e à avaliação do estudante e nas correspondentes práticas, sobretudo nas profissões da saúde ${ }^{3,4}$. Parte substancial dessas mudanças relaciona-se à crescente utilização de estratégias de ensino e aprendizagem mediadas pela tecnologia da informação e comunicação ${ }^{5}$.
Este aumento na utilização dos recursos de "aprendizagem eletrônica" (tradução livre de e-learning ou online learning) foi, mais recentemente, acelerado pela epidemia do novo coronavírus (Covid-19) ${ }^{6,7}$, reconhecida como de escala global em março de 2020. Assim, o que vinha introduzindo-se aos poucos, não sem resistências em muitas instituições, passou a ser a principal estratégia de ensino e aprendizagem a ser utilizada em todas as escolas e em todos os níveis educacionais.

Embora esta pandemia tenha provocado efeitos significativos em praticamente todos os componentes dos processos educacionais e tenha, em muitas escolas das profissões da saúde, inviabilizado completamente as atividades presenciais, um aspecto que foi particularmente afetado foi o da avaliação do estudante ${ }^{8}$. Consequente, torna-se imperioso rever os seus conceitos e suas melhores práticas, em seus aspectos gerais e, especialmente, naqueles que melhor se aplicam a este novo cenário de ensino à distância mediado por recursos de tecnologia.

Neste artigo, apresentamos considerações sobre a avaliação do estudante em condições de educação remota e à distância que julgamos poder ser úteis à comunidade brasileira de educadores nas profissões da saúde. Procuramos compartilhar os conhecimentos e a experiência sobre a avaliação do estudante adquiridos em muitos anos de educação e treinamento profissional, em condições presenciais. A estes acrescem-se o que vivenciamos na utilização constante há vários anos, em menor escala do que a atual, de recursos de aprendizagem eletrônica à distância, como também o que aprendemos no uso intensivo da educação remota ao longo do ano de 2020.

De modo a facilitar a compreensão dos leitores, o texto é dividido em quatro seções que abordam, respectivamente, conceitos básicos de avaliação educacional e da avaliação do estudante, o papel estratégico da avaliação formativa e o valor da devolutiva (feedback), as características da devolutiva efetiva e as correspondentes recomendações para a sua prática e um conjunto de sugestões sobre possíveis maneiras efetivas de praticar a avaliação do estudante na educação remota e à distância, tendo em vista as suas finalidades somativa e formativa. 
CONCEITOS BÁSICOS DE AVALIAÇÃO EDUCACIONAL E DE AVALIAÇÃO DO ESTUDANTE

No campo da educação em geral e na que trata da formação dos profissionais da saúde o conceito de avaliação educacional engloba duas modalidades diferentes, mas complementares, que tem como foco, respectivamente, o estudante e os processos e atividades voltados à aprendizagem. Esta última compreende a "avaliação de programas", entendendo-se por "programa" qualquer conjunto estruturado de atividades voltadas à aprendizagem (unidades ou módulos curriculares, cursos, estágios etc.). A outra modalidade de avaliação educacional é a que tem como objeto o desempenho do estudante. Estas duas modalidades são complementares, mas têm, cada uma, a sua especificidade, por se apoiarem em referenciais teóricos diversos, valer-se de métodos diferentes e, sobretudo, visam à consecução de propósitos distintos ${ }^{9-11}$.

No que tange à avaliação do estudante, embora em muitos contextos ainda se adote o conceito mais restrito de verificação da aprendizagem ou da consecução dos objetivos educacionais previamente definidos, gradualmente disseminase um conceito mais amplo. Nesse conceito mais abrangente, a avaliação do estudante é entendida como processo de obtenção e análise de informações sobre aspectos do seu desempenho que reflitam a aprendizagem, com vistas ao cumprimento de diferentes finalidades ${ }^{1,2,10,11}$. Este conceito é interessante na medida em que os seus componentes remetem a: i) métodos, procedimentos e circunstâncias envolvidas na obtenção de informações; ii) padrões e critérios norteadores da análise das informações obtidas; iii) domínios e componentes do desempenho do estudante; iv) finalidades ou propósitos da avaliação.

A escolha dos métodos deve ser orientada pelos domínios do desempenho que se pretende avaliar. Estes domínios, por sua vez, relacionamse aos objetivos do processo educacional a que a avaliação se refere e devem se associar ao perfil de conhecimentos, habilidades, competências, atributos e comportamentos que se deseja que o estudante apresente como consequência da sua participação no processo educacional. Além disso, a escolha dos métodos de avaliação deve ser informada pela qualidade que cada um deles pode atingir, quando utilizado em boas condições. Esta qualidade é determinada por atributos específicos, como, dentre outros, validade, fidedignidade (confiabilidade), viabilidade e impacto educacional ${ }^{1,11}$

As finalidades e as funções da avaliação do estudante são, possivelmente, a parte mais importante do conceito ampliado antes referido. Quanto à finalidade principal, a avaliação do estudante pode ser classificada em três tipos: somativa, formativa e informativa (ou diagnóstica) (1,2,11. $^{\text {. }}$ A avaliação somativa é aquela que tem como finalidade principal a tomada de decisões que devem afetar em maior ou menor grau o destino do estudante (aprovação, reprovação, classificação entre os melhores, etc. $)^{1,11}$. Interessante mencionar que a palavra somativa (do Inglês summative) significa aquilo que se vale de vários recursos ou indicadores para se chegar a uma determinada conclusão.

A avaliação formativa é a que tem a finalidade principal de contribuir para fomentar a aprendizagem, orientando o(a) estudante, ajudando-o(a) a se conhecer melhor e a fazer planos de aperfeiçoamento ${ }^{1,2,11}$.

A avaliação informativa é aquela que tem como finalidade principal a utilização de dados do desempenho do estudante como indicador da qualidade do processo educativo ${ }^{11}$. Nesta finalidade, os resultados da avaliação do estudante informam a modalidade de avaliação educacional designada "avaliação de programas" 9 . A avaliação informativa é também conhecida como diagnóstica, uma vez que os dados do desempenho do estudante contribuem para o "diagnóstico" da qualidade do ensino. Ressalte-se, porém, que avaliação diagnóstica também designa os procedimentos avaliativos realizados para verificar o grau de domínio das habilidades que se pretende que sejam adquiridas em processo educativo prestes a se iniciar. Coloquialmente, o procedimento utilizado nesta avaliação costuma ser denominado de "préteste", ou seja, o teste que antecede o processo de ensino.

Importante considerar que, mesmo neste milênio onde os progressos nesse campo são imensos, a avaliação do estudante, em muitas 
instituições, resume-se, quase que exclusivamente, à finalidade somativa, cuja prática se traduz na aplicação de exames fortemente voltados ao domínio cognitivo ${ }^{11}$, em geral ao final do desenvolvimento das unidades curriculares, tendo os estudantes sob estrita vigilância para evitar fraudes ("cola") 7 . Por outro lado, vem crescendo o importante movimento de valorização da prática da avaliação formativa, por reconhecer o seu enorme impacto positivo sobre a aprendizagem ${ }^{1,2,11,12}$. Este movimento é mais nítido nos ambientes de treinamento dos programas de formação de profissionais da saúde ${ }^{2,12}$.

O avanço na introdução da aprendizagem eletrônica, mais acelerado com a eclosão da pandemia do Covid-19 veio, por razões diversas, contribuir para a diminuição da importância dos exames de finalidade somativa e para a maior preponderância da avaliação formativa. Deve ser reconhecido, porém, que, em grande parte, esta mudança foi motivada pelas dificuldades operacionais, técnicas e tecnológicas da realização de exames com condições ideais de vigilência? ${ }^{7}$. Essas mesmas dificuldades têm motivado maior preocupação com a necessidade de investimento na educação dos estudantes de modo a desenvolver a sua integridade acadêmica ${ }^{8}$. Deste modo, espera-se que os estudantes evitem conscientemente as fraudes, não porque a vigilância é rigorosa, ou as penas previstas sejam severas, mas pela convicção de que este é um comportamento moralmente inaceitável.

Adicionalmente, no contexto da aprendizagem eletrônica, passam a ser particularmente relevantes conceitos relativos à importância dos exames como recurso específico para fomentar a aprendizagem ("test-enhanced learning"), bem como para evitar o esquecimento induzido por forma inadequada das questões ("test-induced forgetting"), sobretudo no caso dos itens objetivos ("testes de múltipla escolha") ${ }^{13}$, que compatibiliza com práticas próprias da avaliação formativa.

Ainda que muitas das mudanças associadas ao aumento do uso dos recursos de aprendizagem eletrônica se restrinjam ao domínio cognitivo, é intensa a busca de formas de avaliar outros domínios $^{6}$, incluindo as habilidades de comunicação verbal com provas orais ${ }^{14} \mathrm{e}$, mesmo, habilidades clínicas com exames estruturados administrados eletronicamente ${ }^{15}$.

Este conjunto de mudanças sem dúvidas pode contribuir para o estabelecimento de novo paradigma na avaliação do estudante ${ }^{16}$, caracterizado por maior investimento de tempo e de recursos para a avaliação formativa, pela tomada de decisões verdadeiramente "somativas" não somente em função dos resultados de um exame final único, mas de um conjunto maior de indicadores "formativos", obtidos ao longo do desenvolvimento das atividades de ensino e aprendizagem. Assim, a avaliação do estudante será praticada não somente com a finalidade de verificar a aprendizagem ("assessment of learning"), mas de fomentá-las ("assessment for learning"), usando recursos avaliativos eficazes para promover a aprendizagem quando o estudante os utiliza ou a eles se submete ("assessment as learning").

\section{O PAPEL ESTRATÉGICO DA AVALIA- ÇÃO FORMATIVA (EFETIVIDADE) E O PODER DA DEVOLUTIVA (FEEDBACK)}

Em um determinado curso ou disciplina, apesar dos objetivos a serem alcançados pelos estudantes serem os mesmos, nem sempre a trajetória percorrida vai ser igual para todos. Com o advento da pandemia e o aumento da utilização do ensino remoto, a distância física entre o professor e o aluno aumentou, por outro lado, as plataformas utilizadas podem propiciar uma maior oportunidade para interações. Nesse sentido, as avaliações formativas programadas e realizadas frequentemente adquirem um papel estratégico na formação e acompanhamento dos estudantes. Sem avaliações formativas, é muito difícil detectar e oferecer, em tempo adequado, soluções para corrigir eventuais dificuldades enfrentadas pelos estudantes bem como ajustes no conteúdo programático ou mesmo na estrutura curricular. Assim, a avaliação passa a ser uma atividade reguladora do processo de ensino-aprendizagem ${ }^{17}$.

Um dos componentes centrais da avaliação formativa é o feedback ${ }^{18}$ ou devolutiva, considerado, isoladamente, como o mais potente motivador para melhorar o desempenho dos estudantes ${ }^{19}$. O feedback regula o processo de ensino/ 
aprendizagem, auxiliando o estudante a perceber o que ele já alcançou e quanto falta para os objetivos almejados sejam atingidos. Além disso, o feedback ajuda os estudantes a encontrar os caminhos para se aprimorarem e estimula o desenvolvimento da capacidade reflexiva e autoavaliativa dos estudantes ${ }^{17}$.

Diversos estudos demonstraram a importância do feedback no desempenho dos estudantes. Em uma meta-revisão de 2015, os autores demostraram que o feedback apresentava um melhor efeito quando era fornecido frequentemente e como parte de uma estratégia multifacetada. Adicionalmente, algumas características do feedback também o tornavam mais efetivo, entre elas, quando ele era encorajador, específico, bem elaborado e não-ameaçador ${ }^{20}$. Em 2020, uma meta-análise demonstrou que profissionais da saúde que receberam feedback verbal e presencial (face-to-face) apresentavam melhor desempenho nas tarefas, quando comparados com profissionais que não recebiam feedback ${ }^{21}$. Além disso, apesar de, muitas vezes, o feedback ser fornecido presencialmente, alguns estudos já demonstraram a sua efetividade quando oferecido em um ambiente computacional. Interessantemente, muitas características do feedback online se assemelham às do presencial, ou seja, ele é mais efetivo quando fornecido de maneira elaborada e próximo evento ${ }^{22}$.

Nesse sentido, mesmo com todas as mudanças impostas pela pandemia e pelo distanciamento, a avaliação formativa e o feedback permanecem sendo importantes e poderosas ferramentas do processo de ensino-aprendizagem. Porém, é de extrema importância que eles sejam aprimorados e adequados para o ensino à distância.

\section{CARACTERÍSTICAS DA DEVOLUTIVA EFETIVA E RECOMENDAÇÕES PARA A PRÁTICA}

Feedback, especificamente na educação de profissionais da saúde, consiste em fornecer informações específicas ao estudante sobre seu desempenho em determinada atividade, utilizando como base um padrão. O feedback é uma importante estratégia de ensino-aprendizagem cujo principal objetivo é melhorar o desempenho do estudante sendo, portanto, atividade central da avaliação formativa. Uma das principais responsabilidades do corpo docente é garantir que os estudantes adquiram competência para progredir para o próximo nível e, finalmente, para exercer a prática como profissional da saúde ${ }^{23,24 .}$

O feedback promove a aprendizagem do aluno de três maneiras:

- Informa aos estudantes o seu progresso ou falta dele;

- Aconselha os estudantes sobre as necessidades de aprendizagem observadas e recursos disponíveis para facilitar sua aprendizagem; e

- Motiva os estudantes a se engajarem no processo de aprendizagem.

Entretanto, para que o estudante possa aprender a partir do feedback é necessário que este seja fornecido de forma construtiva e positiva, proporcionando ao estudante a reflexão sobre as lacunas do seu aprendizado bem como a oportunidade de ajustar o processo de aquisição conhecimento/habilidades ${ }^{2}$. Esta estratégia também proporciona ao avaliador a oportunidade de obter informações acerca da aprendizagem do estudante e discutir soluções para as dificuldades observadas durante a realização da tarefa pelo estudante.

Para que o feedback seja efetivo, ou seja, realmente funcione como uma estratégia de ensino e aprendizagem ele deve seguir algumas recomendações elencadas a seguir ${ }^{26,27}$.

1. Ambiente respeitoso: é fundamental um clima de confiança e respeito mútuos visto que avaliador e estudante estão trabalhando para que o estudante alcance os resultados esperados ${ }^{28}$. O feedback deve ser uma conversa bidirecional na qual o estudante desempenha um papel importante na avaliação de seu próprio desempenho ${ }^{29}$.

2. Metas e objetivos claros e conhecidos: O estudante deve estar orientado quanto ao ambiente de trabalho, às metas e objetivos da experiência de aprendizagem e às expectativas do seu desempenho durante a experiência ${ }^{28}$. Esta orientação preliminar e discussão de responsabilidades e objetivos definem o cenário e tornam o estudan- 
te mais responsável por seu processo de aprendizagem $^{30}$.

3. Feedback específico: É fundamental que o avaliador indique claramente os comportamentos nos quais o estudante está tendo bom desempenho e aqueles nos quais pode melhorar. Exemplos e revisão dos fatos ocorridos contribuem para que o aluno reflita honestamente sobre seu desempenho. O feedback deve ser baseado no desempenho diretamente observado, e durante o feedba$c k$ corretivo, o tom deve ser respeitoso e de apoio. O foco deve ser em comportamentos que podem ser modificados, não na pessoa ou personalidade ${ }^{31,32}$. Quando os residentes de Medicina Interna foram entrevistados sobre suas percepções de feedback útil, eles sentiram que a devolutiva oportuna e específica era mais eficaz quando acompanhado por sugestões de mudança ${ }^{33}$.

4. Feedback oportuno e regular/constante: Um propósito importante do feedback é formativo, permitindo que o estudante faça as mudanças necessárias antes do final do curso ou unidade de ensino. Se um comportamento precisar de correção, o avaliador deve fornecer feedback o mais rápido possível após o encontro, para que este tenha tempo suficiente para agir. Se o feedback não for fornecido até o final da experiência, o aluno não terá a oportunidade de corrigir o comportamento durante o curso/unidade de ensino. O feedback contínuo e regular é essencial para promover cuidados médicos da mais alta qualidade e satisfação profissional.

5. Induzir o estudante à reflexão: Um objetivo fundamental do treinamento clínico é promover um profissional reflexivo. Estimular o estudante a realizar uma autoavaliação utilizando perguntas abertas para iniciar o feedback pode promover a reflexão do estudante sobre suas práticas. O estudante pode trazer à tona os mesmos pontos que o professor planejou abordar, ajudando a desenvolver o diálogo. O feedback iniciado unicamente ou em conjunto pelos alunos foi visto como mais instrutivo do que aquele iniciado pelos professores ${ }^{31,34}$.
6. Usar estratégias positivas de comunicação: A mensagem enviada pela linguagem corporal é importante; sentado ao lado do estudante pode minimizar uma posição de poder por parte do professor.

7. Limitar o feedback fornecido na sessão ao que $o$ aluno pode absorver ${ }^{30,32}$. Quando o feedback é bem conduzido, pode meIhorar a relação estudante-avaliador e levar a mudanças benéficas no comportamento do estudante (31).

Os professores devem considerar o feedback como qualquer outra habilidade que pode ser adquirida e aprimorada por meio da prática repetida $^{31}$. Vários autores descreveram técnicas para realizar um feedback eficaz. Dentre estas destacam-se as Regras de Pendleton ${ }^{35}$, a técnica sanduiche e a técnica sanduiche modificada (Combo).

Pendleton et al. descreveram um processo de quatro etapas para a realização de uma sessão de feedback. Nesta técnica se inicia perguntando ao estudante o que ele/ela acredita ter realizado adequadamente; o avaliador pode concordar conforme apropriado e adicionar reforçando comentários; segue solicitando ao estudante que o mesmo aponte áreas de melhoria; o avaliador pode concordar conforme apropriado e adicionar mais comentários. A seguir um roteiro prático das Regras de Pendleton ${ }^{35}$.

Passo 1: O que você acha que fez bem....

Passo 2: O que você acha que não foi adequado.

Passo 3: O que eu acho que você fez bem.

Passo 4: 0 que eu acho que você poderia ter feito melhor...

Passo 5: Vamos conversar sobre como poderia melhorar?

$\mathrm{Na}$ técnica sanduiche o feedback sobre comportamentos inadequados é fornecido entre duas ações ou comportamentos positivos. Essa estratégia é particularmente útil para os alunos considerados mais resistentes ${ }^{36}$. A seguir o roteiro descrito nesta técnica:

Passo 1: Comece reconhecendo e reforçando o que o estudante realizou adequadamente. Esta abordagem pode apoiar boas práticas, motivar o estudante a repeti-las e levá-lo a buscar 
mais feedback ${ }^{31,32}$. Os estudantes afirmaram que a devolutiva positiva sobre o que eles estavam realizandocorretamente deu-Ihes confiança em suas habilidades e criou um melhor ambiente de aprendizagem $^{33}$.

Passo 2: Em seguida destaque os pontos que necessitam ser aperfeiçoados com as correções necessárias, fornecendo exemplos e sugestões de melhorias.

Passo 3: Finalize destacando outros pontos positivos. Os alunos relatam que o feedback construtivo foi benéfico, especialmente quando se concentrou no desempenho específico, acompanhado por razões pelas quais o desempenho estava incorreto $^{33}$ e quando se tratava de comportamentos que o aluno foi capaz de controlar ou modificar ${ }^{30}$.

Técnica sanduiche modificada (Combo): Esta técnica acrescenta dois passos muito importantes a técnica anterior, conforme descrito a seguir:

Passo 1: Inicie o feedback convidando o estudante a fazer uma autoavaliação fazendo perguntas abertas para promover a reflexão do estudante sobre suas práticas. Essa autoavaliação pode suavizar a percepção de aspereza e ajudar a tornar o feedback corretivo mais aceitável.

Passo 2: Reconheça e reforce o que o estudante fez adequadamente.

Passo 3: Destaque os pontos que necessitam ser aperfeiçoados com as correções necessárias, fornecendo exemplos e sugestões de melhorias.

Passo 4: Confirme a compreensão do estudante. Uma sessão de feedback pode ser carregada de emoção por parte de avaliador e estudante, especialmente durante o feedback corretivo. É importante aprender sobre as perspectivas do aluno e as possíveis razões para um comportamento específico ${ }^{32}$. Considere a formação, temperamento e disposição do aluno para mudar ${ }^{37}$.

Passo 5: A sessão de feedback deve terminar com um plano de ação para melhorias ${ }^{32,33}$. Solicite ao estudante ideias e auxilie confirmando ou modificando conforme necessário. A participação do estudante na elaboração do plano de melhoria ajudará o mesmo a desenvolver habilidades de reflexão.

Destaca-se que para o constante aperfeiçoamento do feedback é essencial que os avaliadores também reflitam sobre suas habilidades após o término de cada sessão avaliando o que funcio- nou, refletindo sobre o que deve ser modificado e adaptando estratégias para sessões futuras ${ }^{32}$. Fazer da reflexão uma parte de cada encontro de devolutiva vai melhorar as habilidades do professor em dar feedback. Também é importante que os avaliadores tenham espaço para compartilhar experiências e discutir sobre os desafios enfrentados com educadores e colegas com maior experiência. Oficinas e workshops podem enfatizar a aplicação de teorias de aprendizagem de adultos às estratégias de ensino no trabalho e fornecer oportunidades para discussões entre pares e prática de habilidades.

A prática do feedback deve fazer parte da cultura institucional como parte essencial do processo de ensino-aprendizagem ${ }^{38}$. A instituição deve desenvolver um processo de monitoramento do processo de feedback em cursos/ unidades de ensino / rotações individuais ${ }^{37}$. Os professores devem ser solicitados a fornecer feedback aos estudantes e receber feedback destes, dos colegas e de seus superiores sobre seu desempenho como professores.

\section{POSSÍVEIS MANEIRAS EFETIVAS DE PRATICAR A AVALIAÇÃO DO ESTU- DANTE NA ER E NA EAD (FINALIDA- DES SOMATIVA E FORMATIVA)}

Toda vez que se fala de educação se fala de avaliação. Avaliação é uma forma de medir, mensurar, dar objetividade e estabelecer critérios para confirmar se o conteúdo "ensinado" foi, de fato, "aprendido". Na ER e na EaD também existe a busca por métodos de avaliação on-line que permitam medir ou mensurar se os objetivos de estudo foram atingidos ${ }^{39}$. Desta forma, um curso a distância deve desenvolver atividades que possam proporcionar a participação e a construção do conhecimento por parte dos estudantes, viabilizando a prática da avaliação formativa, bem como proporcionar meios que permitam a quantificação como ideologia de medição do conhecimento, viabilizando a prática da avaliação somativa. Tal sistema de avaliação é complexo e despende tempo, pois implica em compreender como o conhecimento sobre o conteúdo trabalhado foi construído e como ele pode ser mensurado 
não só em seus meios (formativo) mas também em seu fim (somativo) ${ }^{40}$.

Por definição, avaliar é produzir um julgamento, uma apreciação. Devemos considerar, no entanto, avaliação como um processo, um compilado de métodos que, ao seu término, permite a maneira mais justa possível estabelecer juízo de valor $^{41}$. Enquanto processo, enquanto "sistema" de avaliação, o ato avaliativo inclui (1) tomada de decisão sobre o que é relevante, (2) registro da informação (testes, provas, exames), (3) interpretação da informação recolhida e, finalmente, (4) o desenvolvimento de uma ação baseada na análise crítica e reflexiva destas informações ${ }^{42}$. Nesse contexto, as três primeiras etapas concretizam a produção de um julgamento, a quarta é consequência das anteriores e resulta no fim definido. Assim, podemos considerar duas modalidades de avaliação: avaliação formativa e avaliação somativa. "A mesma informação, recolhida do mesmo modo, chamar-se-á formativa se for para apoiar a aprendizagem ou somativa se for para registrar ou reportar o conhecimento adquirido" (Harlen, 2005, p. 208). Em resumo podemos atribuir dois grandes objetivos a avaliação: avaliar para ajudar a aprender e avaliar para sintetizar a aprendizagem ${ }^{43}$.

Uma avaliação somativa visa, portanto, a quantificação do conhecimento o que, do ponto de vista educacional, induz o avaliador a uma atribuição de valores ou conceitos ao que o estudante "sabe" ou "aprende". Uma avaliação formativa, por sua vez, tem por objetivo principal o aperfeiçoamento do processo de aprendizagem do estudante. Ou seja, visa apoiar a aprendizagem ${ }^{43}$.

Primeiramente, há uma reflexão sobre o que deve ser avaliado. Nas avaliações somativas, fazer da prova uma imagem especular dos objetivos de estudo propostos é uma maneira de nortear a elaboração da prova, independente do formato do exame. Nas avaliações formativas, esse desafio é maior. Entende-se que, nesta avaliação, a participação, ou seja, a interação do aluno é o que realmente possui maior importância. Neste contexto, o que deve ser avaliado é a participação dos alunos mediante a interação tanto "síncronas" como "assíncronas". Em suma, quando se fala em avaliar do ponto de vista formativo é típico desse tipo de avaliação focar muito mais o processo do que o produto, visando muito mais aperfeiçoar do que medir o que está sendo aprendido ${ }^{42}$.

É natural pensar que o contato entre aluno e professor seja necessário e absoluto para que se possa identificar eventuais mudanças abruptas de comportamento ou fazer real juízo de valor quanto a sua participação. Entretanto, para quem considera o "anonimato" do online como limitante na ER ou na EaD, engana-se. Na ER e na EaD é possível desenvolver formas de avaliação formativa. Como exemplo citamos a descrição/expressão e reflexão/discussão. No caso da ER e na EaD é possível mediante a escrita armazenar registros das 'ideias' dos alunos (descrição/expressão). As ferramentas que podem ser usadas para isso são os portfólios, relatórios de atividades e tarefas em forma de memorandos ou testes específicos. Outras atividades como os fóruns de discussão e as discussões e debates que acontecem em reuniões síncronas permitem avaliar a compreensão do estudante quanto aos assuntos tratados mediante suas propostas e sugestões para resolução de problemas apresentados no debate (reflexão/ discussão). Dessa forma, nos trabalhos apresentados e nesta discussão compartilhada é possível identificar eventuais "erros" conceituais e, mediante feedback, sanear eventuais fragilidades ao mesmo tempo que é possível favorecer e destacar eventuais fortalezas ${ }^{44}$.

A exemplo das atividades presenciais, qualquer avaliação formativa traz em si os mesmos desafios na ER e na EaD. Ao avaliar interações, o pensar e, principalmente, a construção do conhecimento, faz-se um processo de grande subjetividade quando queremos atribuir valores quantitativos a esses aspectos. Mas não podemos perder o referencial de que avaliar também deve ser, antes de tudo, uma orientação para o professor na condução de sua prática docente. Compreender como o aluno está desenvolvendo conceitos e aperfeiçoando práticas é fundamental para qualquer forma de ensino aprendizagem quer seja presencial ou remota ${ }^{45}$.

Para validar a avaliação no sentido de determinar ou verificar se o conteúdo ensinado foi de fato aprendido, temos a avaliação somativa. Como já foi dito, a avaliação somativa é aquela que tem como finalidade principal a tomada de decisões que devem afetar em maior ou menor 
grau o destino do estudante (aprovação, reprovação, classificação entre os melhores etc.). Para tanto, esse processo de avaliação pode se valer de vários recursos ou indicadores para se chegar a uma determinada conclusão. Entre eles: prova oral, prova prática, teste de conhecimento com questões de múltipla escolha, teste de conhecimento com questões dissertativas, ou abertas, ensaios clínicos, etc. A exemplo da educação presencial, inclusive para algumas habilidades (prova prática), todas estas modalidades podem ser desenvolvidas na ER e na EaD. Plataformas específicas, ferramentas digitais e novas tecnologias estão disponíveis para aplicação de testes, provas, quis, de forma segura garantindo a confiabilidade da avaliação. Formas práticas de avaliação mediante a gravação de vídeos, ou prova teórica mediante discussões e arguições que podem ser inclusive gravadas servem não só a avaliações somativas mas também formativas ${ }^{46}$.

O proctoring, também chamada de 'fiscalização de exames', é um método de garantir segurança da prova realizada em caráter remoto ou online. Inclui fiscalização dos alunos durante a realização de testes, exames ou questionários. O sistema proctoring é usada em uma variedade de contextos, incluindo ensino primário, secundário e superior, bem como ambientes de teste padronizados, e alguns exames de credenciamento profissional. O proctoring avalia e monitora o comportamento do estudante durante os exames administrados eletronicamente, identifica sinais de quebra de sigilo como conversas paralelas, desvio significativo do olhar, presença de terceiros no local do exame, cenário e violação do sistema eletrônico ou plataforma que a prova está sendo realizada. O proctoring auxilia na segurança da prova e do processo de avaliação tornando possível esta etapa do ensino-aprendizagem na ER e na EaD ${ }^{47}$.

Durante a pandemia de COVID-19 de 2020 muitas universidades voltaram-se para plataformas de e-proctoring. Diante desta nova realidade, estudantes em todo o mundo questionaram o uso de serviços comerciais de e-proctoring em suas instituições de ensino superior, produto de uma desconfiança natural a uma "nova" prática de avaliação sem a presença física do professor.
Apesar dessa resistência inicial e familiarizados com o uso da tecnologia no seu dia a dia e também com a ER e a EaD, os estudantes foram se dessensibilizando, se permitiram conhecer esta ferramenta e compreender este método como seguro e exequível.

Se entendemos avaliação formativa como uma forma de aperfeiçoar o processo de aprendizagem, temos a figura do professor como indispensável neste processo quer seja de forma remota ou presencial. Por sua vez, se entendemos avaliação somativa como uma exigência realizada pelo sistema educacional que incita uma atribuição de valores baseado na "medição" do conhecimento adquirido, esta poderia ser transferida para a instituição. Deixar o professor mais com a avaliação formativa bem feita e deixar a somativa, que leva a tomadas de decisão para a instituição fazer em momentos específicos, utilizando esses recursos mais adequados e de maior custo seria uma forma ergonômica e justa de organizar e pensar um sistema de avaliação de forma até programática.

Quando se fala em ensino-aprendizagem, naturalmente se imagina atividades presenciais. Da mesma forma, quando se fala em avaliação, naturalmente se imagina provas e exames de caráter somativo. Considerar estratégias de avaliação formativa é sempre um esforço que demanda tempo e planejamento maior por parte de qualquer educador. Repensar a educação e a avaliação em caráter remoto e na EaD é um desafio ainda maior, mas absolutamente possível.

\section{REFERÊNCIAS}

1. Panúncio-Pinto MP, Troncon LEA. Avaliação do estudante - aspectos gerais. Medicina (Ribeirão Preto). 2014;47(3):314-23.

2. Borges MC, Miranda CH, Santana RC, Bollela VR. Avaliação fomativa e feedback como ferramenta de aprendizado na formação de profissionais de saúde. Medicina (Ribeirão Preto). 2014;47(3):324-31

3. Norcini J, Anderson MB, Bollela V, Burch V, Costa MJ, Duvivier R, et al. 2018 Consensus framework for good assessment. Med Teach. 2018;40(11):1102-9.

4. Norcini J, Anderson B, Bollela V, Burch V, Costa MJ, Duvivier $R$, et al. Criteria for good assessment: consensus statement and recommendations from the Ottawa 2010 Conference. Med Teach. 2011;33(3):206-14. 
5. Conrad D, Openo J. Assessment strategies for online learning: Engagement and authenticity. Edmonton, Alberta, Canada: AU Press; 2018.

6. Gordon $M$, Patricio M, Horne L, Muston A, Alston SR, Pammi $M$, et al. Developments in medical education in response to the COVID-19 pandemic: A rapid BEME systematic review: BEME Guide No. 63. Med Teach. 2020;42(11):1202-15.

7. Butler-Henderson K, Crawford J. A systematic review of online examinations: A pedagogical innovation for scalable authentication and integrity. Comput Educ. 2020;159:104024.

8. Rahim AFA. Guidelines for Online Assessment in Emergency Remote Teaching during the COVID-19 Pandemic. Education in Medicine Journal. 2020;12(2):59-68.

9. Bollela VR, Castro M. Avaliação de programas educacionais nas profissões da saúde: conceitos básicos. Medicina (Ribeirao Preto). 2014;47(3):332-42.

10. Vianna HM. Introdução à avaliação educacional. São Paulo: IBRASA. 1989.

11. Troncon LEA. Estruturação de Sistemas para Avaliação Programática do Estudante de Medicina. Revista Brasileira de Educação Médica. 2016;1:30-42

12. Norcini J, Burch V. Workplace-based assessment as an educational tool: AMEE Guide No. 31. Med Teach. 2007;29(9):855-71.

13. Little JL, Bjork EL, Bjork RA, Angello G. Multiple-choice tests exonerated, at least of some charges: fostering test-induced learning and avoiding test-induced forgetting. Psychol Sci. 2012;23(11):1337-44.

14. Akimov A, Malin M. When old becomes new: a case study of oral examination as an online assessment tool. Assessment \& Evaluation in Higher Education. 2020;45(8):1205-21

15. Hannon P, Lappe K, Griffin C, Roussel D, Colbert-Getz J. An objective structured clinical examination: From examination room to Zoom breakout room. Med Educ. 2020;54(9):861

16. Harden RM. Ten key features of the future medical school-not an impossible dream. Med Teach. 2018;40(10):1010-5.

17. Swanwick T. Understanding Medical Education: Evidence, Theory and Practice: Wiley-Blackwell; 2010.

18. Rushton A. Formative assessment: a key to deep learning? Med Teach. 2005;27(6):509-13.

19. Hattie J. Measuring the Effects of Schooling. Australian Journal of Education. 1992;36(1):5-13.

20. van de Ridder JM, McGaghie WC, Stokking KM, ten Cate OT. Variables that affect the process and outcome of feedback, relevant for medical training: a meta-review. Med Educ. 2015;49(7):658-73.

21. Johnson CE, Weerasuria MP, Keating JL. Effect of faceto-face verbal feedback compared with no or alternative feedback on the objective workplace task performance of health professionals: a systematic review and meta -analysis. BMJ Open. 2020;10(3):e030672.

22. Van der Kleij FM, Feskens RCW, Eggen TJHM. Effects of Feedback in a Computer-Based Learning Environment on Students' Learning Outcomes: A Meta-Analysis. Review of Educational Research. 2015;85(4):475-511.

23. Hauer KE, Holmboe ES, Kogan JR. Twelve tips for implementing tools for direct observation of medical trainees' clinical skills during patient encounters. Med Teach. 2011;33(1):27-33.

24. van de Ridder JM, Stokking KM, McGaghie WC, ten Cate OT. What is feedback in clinical education? Med Educ. 2008;42(2):189-97.

25. Gibbs G. Using assessment strategically to change the way students learn. In: Brown S, Glasner A, editors. Assessment Matters in Higher Education: Choosing and Using Diverse Approaches: Open University Press; 1999.

26. Zeferino $\mathrm{AMB}$, Domingues $\mathrm{RCL}$, Amaral E. Feedback como estratégia de aprendizado no ensino médico. Rev bras educ méd. 2007;31(2):176-9.

27. Ramani S. Twelve tips to improve bedside teaching. Med Teach. $2003 ; 25(2): 112-5$.

28. Hewson MG, Little ML. Giving feedback in medical education: verification of recommended techniques. J Gen Intern Med. 1998;13(2):111-6.

29. Krackov SK. Expanding the horizon for feedback. Med Teach. 2011;33(11):873-4.

30. Wood BP. Feedback: a key feature of medical training. Radiology. 2000;215(1):17-9.

31. Cantillon P, Sargeant J. Giving feedback in clinical settings. BMJ. 2008;337:a1961.

32. Krackov SK. Giving feedback. In: Dent JA, Harden RM, editors. A Practical Guide for Medical Teachers. 3rd ed: Elsevier; 2009.

33. Bing-You RG, Paterson J, Levine MA. Feedback falling on deaf ears: residents' receptivity to feedback tempered by sender credibility. 1997;19(1):40-4.

34. Van Hell EA, Kuks JB, Raat AN, Van Lohuizen MT, Cohen-Schotanus J. Instructiveness of feedback during clerkships: influence of supervisor, observation and student initiative. Med Teach. 2009;31(1):45-50.

35. Pendleton D, Scofield T, Tate P, Havelock P. The Consultation: An Approach to Learning and Teaching. Oxford: Oxford University Press; 1984.

36. Lucas JH, Stallworth JR. Providing difficult feedback: TIPS for the problem learner. Fam Med. 2003;35(8):544-6.

37. Milan FB, Dyche L, Fletcher J. "How am I doing?" Teaching medical students to elicit feedback during their clerkships. Med Teach. 2011;33(11):904-10.

38. Krackov SK, Pohl H. Building expertise using the deliberate practice curriculum-planning model. Med Teach. $2011 ; 33(7): 570-5$

39. Rocha HV. O ambiente TelEduc para educação a distância baseada na web: Princípios, funcionalidades e perspectivas de desenvolvimento. In: Moraes MC, editor. Edu- 
cação a distância: fundamentos e práticas. Campinas: UNICAMP/NIED; 2002. p. 197-212.

40. Rosa M, Maltempi MM. A avaliação vista sob o aspecto da educação a distância. Ensaio: Avaliação e Políticas Públicas em Educação. 206;14:57-75.

41. Bennett RE. Formative assessment: a critical review. Assessment in Education: Principles, Policy \& Practice. $2011 ; 18(1): 5-25$.

42. Santos L. A articulação entre a avaliação somativa e a formativa, na prática pedagógica: uma impossibilidade ou um desafio? Ensaio: Avaliação e Políticas Públicas em Educação. 2016;24(637):637-69

43. Harlen W. Teachers' summative practices and assessment for learning - tensions and synergies. The Curriculum Journal. 2007;16(2):207-23.
44. Harlen W, James M. Assessment and Learning: differences and relationships between formative and summative assessment. Assessment in Education: Principles, Policy \& Practice. 2006;4(3):365-79.

45. Gaur U, Majumder MAA, Sa B, Sarkar S, Williams A, Singh K. Challenges and Opportunities of Preclinical Medical Education: COVID-19 Crisis and Beyond. SN Compr Clin Med. 2020:1-6.

46. Kenski VM. Tecnologias e ensino presencial e a distância: Papirus Editora; 2003.

47. Dragan IF, Yildiz LF, Dunn K, Ramesh A. Integrating remote proctoring in dental education: Problem, solution, and results. J Dent Educ. 2020 Jul 11. doi: 10.1002/ jdd. 12307.

Editor:

Prof. Dr. Marcelo Riberto

Recebido: $30 / 04 / 2021$

Aprovado: 13/07/2021

cc) (i) Este é um artigo publicado em acesso aberto (Open Access) sob a licença Creative 